\title{
Lowered stomata conductance promotes the oxidative burst, an essential factor in the promotion of programmed cell death Per Mühlenbock* and Stanislaw Karpinski
}

\author{
Address: Department of Botany, Stockholm University, Stockholm, SE-10691, Sweden \\ Email: Per Mühlenbock* - Per.Muhlenbock@botan.su.se \\ * Corresponding author
}

from Cell Biology of Nitric Oxide and Cell Death in Plants

Yalta, Ukraine, 8-II September 2004

Published: 3I May 2005

BMC Plant Biology 2005, 5(SuppI I):S24 doi:I0.I I86/I47I-2229-5-SI-S24

The role of stomata in photosynthesis and their importance in plant productivity is well established and a number of studies have shown their importance for the plant's interaction with the abiotic environment. However an increasing amount of evidence is pointing toward an important role for stomata also in biotic defense responses. Using programmed cell death mutants and pathogen infection we set out to investigate the role of stomata in biotic stress. Physiological characterization of the lsd1 mutant of Arabidopsis thaliana revealed a correlation between stomata conductance, $\mathrm{H}_{2} \mathrm{O}_{2}$ production and the spreading of cell death. When challenging wild type plants with the bacterial pathogen Pseudomonas syringae, stomata closed and limiting gas exchange strongly enhanced the spreading of cell death both in infected plants and in the $l s d 1$ mutant. Further studies showed that limiting gas exchange enhances the production of reactive oxygen species that lead to the formation of focused hypersensitive response like lesions in ws-0. Our results clearly indicate the importance of stomata regulation in the spreading of cell death. 